\title{
The uniform and pointwise estimates for polynomials on the weighted Lebesgue spaces in the general regions of complex plane
}

\author{
P. Özkartepe ${ }^{1}$, F.G. Abdullayev*2,3 \\ ${ }^{1}$ Gaziantep University Education Faculty in Nizip Department of Mathematics and Science Education, \\ Nizip/Gaziantep-TURKEY. \\ ${ }^{2}$ Kyrgyz-Turkish Manas University, Faculty of Science, Department of Applied Mathematics and \\ Informatics, 720044 Bishkek-KYRGYZ REPUBLIC. \\ ${ }^{3}$ Mersin University, Faculty of Arts and Science, Department of Mathematics, 33343 Mersin-TURKEY.
}

\begin{abstract}
The estimation of the modulus of algebraic polynomials on the boundary contour with weight function, having some singularities, with respect to the their quasinorm, on the weighted Lebesgue space was studied in this current work.
\end{abstract}

Mathematics Subject Classification (2010). Primary 30A10, 30C10; Secondary $41 \mathrm{~A} 17$

Keywords. algebraic polynomials, conformal mapping, quasicircle

\section{Introduction}

Let $\mathbb{C}$ be a complex plane, and $\overline{\mathbb{C}}:=\mathbb{C} \cup\{\infty\} ; G \subset \mathbb{C}$ be the bounded Jordan region, with $0 \in G$ and the boundary $L:=\partial G$ be a closed Jordan curve, $\Omega:=\overline{\mathbb{C}} \backslash \bar{G}=\operatorname{ext} L$. Let $\wp_{n}$ denotes the class of arbitrary algebraic polynomials $P_{n}(z)$ of degree at most $n \in \mathbb{N}:=$ $\{1,2, \ldots\}$.

Let $0<p \leq \infty$. For a rectifiable Jordan curve $L$, we denote

$$
\begin{aligned}
& \left\|P_{n}\right\|_{\mathcal{L}_{p}}:=\left\|P_{n}\right\|_{\mathcal{L}_{p}(h, L)}:=\left(\int_{L} h(z)\left|P_{n}(z)\right|^{p}|d z|\right)^{1 / p}, 0<p<\infty ; \\
& \left\|P_{n}\right\|_{\mathcal{L}_{\infty}}:=\left\|P_{n}\right\|_{\mathcal{L}_{\infty}(1, L)}:=\max _{z \in L}\left|P_{n}(z)\right|, p=\infty .
\end{aligned}
$$

Clearly, $\|\cdot\|_{\mathcal{L}_{p}}$ is the quasinorm (i.e. a norm for $1 \leq p \leq \infty$ and a $p$-norm for $0<p<1$ ).

Denoted by $w=\Phi(z)$, the univalent conformal mapping of $\Omega$ onto $\Delta:=\{w:|w|>1\}$ with normalization $\Phi(\infty)=\infty, \lim _{z \rightarrow \infty} \frac{\Phi(z)}{z}>0$ and $\Psi:=\Phi^{-1}$. For $t \geq 1$ we set

$$
L_{t}:=\{z:|\Phi(z)|=t\}, L_{1} \equiv L, G_{t}:=\operatorname{int} L_{t}, \Omega_{t}:=\operatorname{ext} L_{t} .
$$

*Corresponding Author.

Email addresses: pelinozkartepe@gmail.com (P. Özkartepe), fabdul@mersin.edu.tr;

fahreddinabdullayev@gmail.com (F.G. Abdullayev)

Received: 17.11.2016; Accepted: 19.09.2017 
Let $\left\{z_{j}\right\}_{j=1}^{m}$ be a fixed system of distinct points on curve $L$ which is located in the positive direction. For some fixed $R_{0}, 1<R_{0}<\infty$, and $z \in G_{R_{0}}$, consider a so-called generalized Jacobi weight function $h(z)$ being defined as follows

$$
h(z):=h_{0}(z) \prod_{j=1}^{m}\left|z-z_{j}\right|^{\gamma_{j}},
$$

where $\gamma_{j}>-1$, for all $j=1,2, \ldots, m$, and $h_{0}$ is uniformly separated from zero in $G_{R_{0}}$, i.e. there exists a constant $c_{0}:=c_{0}\left(G_{R_{0}}\right)>0$ such that for all $z \in G_{R_{0}}$

$$
h_{0}(z) \geq c_{0}>0 .
$$

In many areas, we often need to study the following inequality

$$
\left\|P_{n}\right\|_{\mathcal{L}_{q}(h, L)} \leq c \mu_{n}(L, h, p, q)\left\|P_{n}\right\|_{\mathcal{L}_{p}(h, L)}, 0<p<q \leq \infty,
$$

where $c=c(G, p, q)>0$ is the constant, independent from $n, P_{n}$, and $\mu_{n}(L, h, p, q) \rightarrow \infty$, $n \rightarrow \infty$, depending on the geometrical properties of curve $L$ and weight function $h$ in the neighborhood of the points $\left\{z_{j}\right\}_{j=1}^{m}$. In particular, it was studied the behavior of the $\left|P_{n}(z)\right|$ on $L(q=\infty)$, where the boundary curve $L$ and weight function $h$ having some singularity on the $L$. First result of the (1.2)-type, in case of $h(z) \equiv 1$ and $L=\{z:|z|=1\}$ for $0<p<\infty$ was found in [13]. The other results, similar to (1.2), for the sufficiently smooth curve, were obtained in [25] $(h(z) \equiv 1)$, and in [27] $(h(z) \neq 1)$. The estimations of the (1.2)-type for $0<p<\infty$ and for $h(z) \equiv 1$ when $L$ is a rectifiable Jordan curve were investigated in [16], [17], [19, pp.122-133], [23], [26] and for $h(z) \neq 1$ - in [10, Theorem $6],[1,2,4-8]$ and others, respectively. More references regarding the inequality of the (1.2)-type can also be found cited above and in Milovanovic et al. [18, Sect.5.3].

Let a rectifiable Jordan curve $L$ has a natural parametrization $z=z(s), 0 \leq s \leq l:=$ mesL. It is said that $L \in C(1, \lambda), 0<\lambda<1$, if $z(s)$ is continuously differentiable and $z^{\prime}(s) \in \operatorname{Lip} \lambda$. Let $L$ belongs to $C(1, \lambda)$ everywhere except for a single point $z_{1} \in L$, i.e. the derivative $z^{\prime}(s)$ satisfies the Lipschitz condition on the $[0, l]$ and $z(0)=z(l)=z_{1}$, but $z^{\prime}(0) \neq z^{\prime}(l)$. It is assumed that $L$ has a corner at $z_{1}$ with exterior angle $\nu_{1} \pi, 0<\nu_{1} \leq 2$, and denoted the set of such curves by $C\left(1, \lambda, \nu_{1}\right)$.

In [28], this problem was investigated in case $p=2$ for orthonormal on the curve of $L \in C\left(1, \lambda, \nu_{1}\right)$ polynomials $Q_{n}(z)$ with the weight function $h$ defined in (1.1) and can be shown that the condition of "pay off" singularity curve and weight function at the points $z_{1}$ can be given as $\left(1+\gamma_{1}\right) \nu_{1}=1$. In [28], the case, where $\left(1+\gamma_{1}\right) \nu_{1} \neq 1$ was investigated by the author. It is shown, that if the singularity of a curve and weight function at the points $z_{1}$ satisfy the following condition

$$
\left(1+\gamma_{1}\right) \nu_{1}<1
$$

then for $\left|Q_{n}(z)\right|$, the following estimation is true

$$
\left|Q_{n}(z)\right| \leq c(L)\left(n^{s_{1}}+\left|z-z_{1}\right|^{\sigma_{1}} \sqrt{n}\right), z \in L,
$$

where

$$
s_{1}=\frac{1}{2}\left(1+\gamma_{1}\right) \nu_{1}, \sigma_{1}=\frac{1}{2}\left(\frac{1}{\nu_{1}}-1-\gamma_{1}\right),
$$

and $c(L)>0$ is the constant independent on $n$.

In this work, the estimations of the (1.2)-type, in particular (1.4)-type for more general curves of the complex plane were studied and the analog of the estimate (1.4) under the condition (1.3) was obtained. 


\section{Definitions and main results}

Throughout this paper, $c, c_{0}, c_{1}, c_{2}, \ldots$ are positive and $\varepsilon_{0}, \varepsilon_{1}, \varepsilon_{2}, \ldots$ are sufficiently small positive constants (generally, different in different relations), depending on $G$ in general and on parameters inessential for the argument. Otherwise, such dependence will be explicitly stated.

Without loss of generality, the number $R_{0}$ in the definition of the weight function, $R_{0}=2$ can be taken. Otherwise, the number $n$ can be chosen as $n \geq\left[\frac{\varepsilon_{0}}{R_{0}-1}\right]$, where $\varepsilon_{0}$, $0<\varepsilon_{0}<1$, some fixed small constant.

For any $k \geq 0$ and $m>k$, notation $i=\overline{k, m}$ means $i=k, k+1, \ldots, m$.

Before go any further, some definitions and the notations are needed to be given. Let $z=\psi(w)$ be the univalent conformal mapping of $B:=\{w:|w|<1\}$ onto the $G$ normalized by $\psi(0)=0, \psi^{\prime}(0)>0$.

By [21, pp.286-294], it is said that, a bounded Jordan region $G$ is called a $\kappa$-quasidisk, $0 \leq \kappa<1$, if any conformal mapping $\psi$ can be extended to a $K$-quasiconformal, $K=\frac{1+\kappa}{1-\kappa}$, homeomorphism of the plane $\overline{\mathbb{C}}$ on the plane $\overline{\mathbb{C}}$. In that case, the curve $L:=\partial G$ is called a $\kappa$-quasicircle. The region $G$ (curve $L$ ) is called a quasidisk (quasicircle), if it is $\kappa$-quasidisk ( $\kappa$-quasicircle) for some $0 \leq \kappa<1$.

We denote the class of $\kappa$-quasicircle by $Q(\kappa), 0 \leq \kappa<1$, and say that $L \in Q$, if $L \in Q(\kappa)$, for some $0 \leq \kappa<1$.

It is well-known that, the quasicircle may not even be locally rectifiable [14, p.104].

Definition 2.1. We say that $L \in \widetilde{Q}, 0 \leq \kappa<1$, if $L$ is a quasicircle and rectifiable.

Definition 2.2. We say that $L \in Q_{\alpha}, 0<\alpha \leq 1$, if $L$ is a quasicircle and $\Phi \in$ Lip $\alpha$, $z \in \bar{\Omega}$.

We note that the class $Q_{\alpha}$ is sufficiently wide. A detailed information on this and the related topics are contained in [15], [22], [30] (see also the references cited therein). We consider only some cases:

Remark 2.3. a) If $L=\partial G$ is a Dini-smooth curve [22, p.48], then $L \in Q_{1}$.

b)If $L=\partial G$ is a piecewise Dini-smooth curve and largest exterior angle at $L$ has opening $\alpha \pi, 0<\alpha \leq 1$, [22, p.52], then $L \in Q_{\alpha}$.

c)If $L=\partial G$ is a smooth curve having continuous tangent line, then $L \in Q_{\alpha}$ for all $0<\alpha<1$.

d) If $L$ is quasismooth (in the sense of Lavrentiev), that is, for every pair $z_{1}, z_{2} \in$ $L, s\left(z_{1}, z_{2}\right)$ represents the smallest of the lengths of the arcs joining $z_{1}$ to $z_{2}$ on $L$, there exists a constant $c>1$ such that $s\left(z_{1}, z_{2}\right) \leq c\left|z_{1}-z_{2}\right|$, then $\Phi \in \operatorname{Lip} \alpha$ for $\alpha=$ $\frac{1}{2}\left(1-\frac{1}{\pi} \arcsin \frac{1}{c}\right)^{-1}[30]$.

e) If $L$ is "c-quasiconformal" (see, for example, [15]), then $\Phi \in \operatorname{Lip} \alpha$ for $\alpha=$ $\frac{\pi}{2\left(\pi-\arcsin \frac{1}{c}\right)}$. Also, if $L$ is an asymptotic conformal curve, then $\Phi \in \operatorname{Lip} \alpha$ for all $0<$ $\alpha<1[15]$.

Definition 2.4. We say that $L \in \widetilde{Q}_{\alpha}, 0<\alpha \leq 1$, if $L \in Q_{\alpha}$ and $L$ is rectifiable.

In this case the following can be had:

Theorem A. ([20]) Let $p>0$. Suppose that $L \in \widetilde{Q}_{\alpha}$, for some $0<\alpha \leq 1$ and $h(z)$ defined as in (1.1) with $\gamma_{j}=0$, for all $j=\overline{1, m}$. Then, for any $P_{n} \in \wp_{n}, n \in \mathbb{N}$, there exists $c_{1}=c_{1}(L, p)>0$ such that

$$
\left\|P_{n}\right\|_{\mathcal{L}_{\infty}} \leq c_{1}\left\|P_{n}\right\|_{\mathcal{L}_{p}\left(h_{0}, L\right)} \begin{cases}n^{\frac{1}{\alpha p}}, & \frac{1}{2} \leq \alpha \leq 1, \\ n^{\frac{\delta}{p}}, & 0<\alpha<\frac{1}{2},\end{cases}
$$

where $\delta=\delta(L), \delta \in[1,2]$, is a certain number. 
Therefore, according to 2.3, $\alpha$ can be calculated in the right parts of estimation (2.1) for each case, respectively.

Now, we assume that the weight function $h$ have "singularity" at the points $\left\{z_{i}\right\}_{i=1}^{m}$, i.e., $\gamma_{i} \neq 0$ for all $i=\overline{1, m}$. In this case, the following "local" (at the points $\left\{z_{i}\right\}_{i=1}^{m} \in L$ ) estimation is holds:

Theorem 2.5. Let $p>0$. Suppose that $L \in \widetilde{Q}_{\alpha}$, for some $\frac{1}{2} \leq \alpha \leq 1$ and $h(z)$ defined as in (1.1). Then, for any $\gamma_{i}>-1, i=\overline{1, m}$, and $P_{n} \in \wp_{n}, n \in \mathbb{N}$, there exists $c_{2}=$ $c_{2}\left(L, p, \gamma_{i}, \alpha\right)>0$ such that

$$
\left|P_{n}\left(z_{i}\right)\right| \leq c_{2} n^{\frac{\gamma_{i}+1}{\alpha p}}\left\|P_{n}\right\|_{\mathcal{L}_{p}(h, L)} .
$$

The inequality (2.2) is sharp. For the polynomial $P_{n}^{*}(z)=1+z+\ldots+z^{n}, h^{*}(z)=h_{0}(z)$, $h^{* *}(z)=|z-1|^{\gamma}, \gamma>0, L:=\{z:|z|=1\}$ and any $n \in \mathbb{N}$, there exists $c_{3}=c_{3}\left(h^{*}, p\right)>0$ ,$c_{3}^{\prime}=c_{3}^{\prime}\left(h^{* *}, p\right)>0$ such that

$$
\begin{aligned}
& \text { a) }\left\|P_{n}^{*}\right\|_{\mathcal{L}_{\infty}} \geq c_{3} n^{\frac{1}{p}}\left\|P_{n}^{*}\right\|_{\mathcal{L}_{p}\left(h^{*}, L\right)}, \quad p>1 ; \\
& \text { b) }\left\|P_{n}^{*}\right\|_{\mathcal{L}_{\infty}} \geq c_{3}^{\prime} n^{\frac{\gamma+1}{p}}\left\|P_{n}^{*}\right\|_{\mathcal{L}_{p}\left(h^{* *}, L\right)}, \quad p>\gamma+1 .
\end{aligned}
$$

Let's introduce the special "singular" points on the curve $L$ and then lets give the following definition. For $\delta>0$ and $z \in \mathbb{C}$, let us set $B(z, \delta):=\{\zeta:|\zeta-z|<\delta\}, \Omega(z, \delta):=$ $\Omega \cap B(z, \delta)$.

Definition 2.6. We say that $L \in Q_{\alpha, \beta_{1}, \ldots, \beta_{m}}, 0<\beta_{i} \leq \alpha \leq 1, i=\overline{1, m}$, if

i) for every sequence non-crossing in pairs circles $\left\{D\left(\zeta_{i}, \delta_{i}\right)\right\}_{i=1}^{m}$ restriction of the function $\Phi$ on $\Omega\left(\zeta_{i}, \delta_{i}\right)$ belongs to $\operatorname{Lip} \beta_{i}\left(\Phi \mid \Omega\left(\zeta_{i}, \delta_{i}\right) \in \operatorname{Lip} \beta_{i}\right)$, and restriction

$$
\Phi \mid \Omega \backslash \bigcup_{i=1}^{m} \Omega\left(\zeta_{i}, \delta_{i}\right) \in \operatorname{Lip} \alpha,
$$

ii) there exists a sequence non-crossing in pairs circles $\left\{D\left(\zeta_{i}, \delta_{i}^{*}\right)\right\}_{i=1}^{m}$, such that $\forall i=\overline{1, m}$, $\delta_{i}^{*}>\delta_{i}$ and $\forall \xi, z \in \Omega\left(\zeta_{i}, \delta_{i}^{*}\right), z \neq \zeta_{i} \neq \xi$ is fulfilled estimation

$$
|\Phi(z)-\Phi(\xi)| \leq k_{i}(z, \xi)|z-\xi|^{\alpha},
$$

where

$$
k_{i}(z, \xi)=c_{i} \max \left(\left|\xi-\zeta_{i}\right|^{\beta_{i}-\alpha} ;\left|z-\zeta_{i}\right|^{\beta_{i}-\alpha}\right),
$$

and $c_{i}$ are independed on $z$ and $\xi$.

Definition 2.7. We say that $L \in \widetilde{Q}_{\alpha, \beta_{1}, \ldots, \beta_{m}}, 0<\beta_{i} \leq \alpha \leq 1, i=\overline{1, m}$, if $L \in$ $Q_{\alpha, \beta_{1}, \ldots, \beta_{m}}, 0<\beta_{i} \leq \alpha \leq 1, i=\overline{1, m}$ and $L=\partial G$ is rectifiable.

It is clear that, from Definition $2.6(2.7)$, each region $L \in \widetilde{Q}_{\alpha, \beta_{1}, \ldots, \beta_{m}}, 0<\beta_{i} \leq \alpha \leq$ $1, i=\overline{1, m}$, may have the "singularity"at the points $\left\{\zeta_{i}\right\}_{i=1}^{m} \in L$. If the region $L$ does not have such "singularity", i.e. if $\beta_{i}=\alpha$, for all $i=\overline{1, m}$, then, it can be written as $G \in \widetilde{Q}_{\alpha}, 0<\alpha \leq 1$.

Throughout this work, we will assume that the points $\left\{z_{i}\right\}_{i=1}^{m} \in L$ defined in (1.1), and $\left\{\zeta_{i}\right\}_{i=1}^{m} \in L$ defined in Definitions 2.6, and 2.7 are coincide. Without loss of generality, the points $\left\{z_{i}\right\}_{i=1}^{m}$ ordered in the positive direction on the curve $L$.

We assume that the curve $L$ has "singularity" on the boundary points $\left\{z_{i}\right\}_{i=1}^{m}$, i.e., $\beta_{i} \neq \alpha$, for all $i=\overline{1, m}$, and the weight function $h$ has "singularity" at the same points, i.e., $\gamma_{i} \neq 0$ for some $i=\overline{1, m}$. In [20], the following result was shown: 
Theorem B. Let $p>0$. Suppose that $L \in \widetilde{Q}_{\alpha, \beta_{1}, \ldots, \beta_{m}}$, for some $\frac{1}{2} \leq \beta_{i} \leq \alpha \leq 1, i=\overline{1, m}$ and $h(z)$ defined as in (1.1) and

$$
\gamma_{i}+1=\frac{\beta_{i}}{\alpha}
$$

for each points $\left\{z_{i}\right\}_{i=1}^{m}$. Then, for any $P_{n} \in \wp_{n}, n \in \mathbb{N}$, there exists $c_{4}=c_{4}\left(L, p, \gamma_{i}, \alpha\right)>0$ such that

$$
\left\|P_{n}\right\|_{\mathcal{L}_{\infty}} \leq c_{4} n^{\frac{1}{\alpha p}}\left\|P_{n}\right\|_{\mathcal{L}_{p}(h, L)} .
$$

In comparisons of Theorem B and Theorem 2.5, it is seen that, if interference conditions (2.4) are satisfied for any "singular" points $\left\{z_{i}\right\}_{i=1}^{m}$ of the weight function and the curve $L$, then the growth rate of the polynomials $P_{n}(z)$ on $L$ does not depend on whether or not the weight function $h(z)$ and the curve $L$.

Now, assume that the equality (2.4) does not hold for each singular points $\left\{z_{i}\right\}_{i=1}^{m}$. Let us suppose

$$
\gamma_{i}+1 \neq \frac{\beta_{i}}{\alpha}
$$

for each singular point $\left\{z_{i}\right\}_{i=1}^{m}$. In the present paper the case is investigated

$$
\gamma_{i}+1<\frac{\beta_{i}}{\alpha}
$$

For simplicity of our next calculations, will be taken as $i=1$.

Theorem 2.8. Let $p>0$. Suppose that $G \in \widetilde{Q}_{\alpha, \beta_{1}}$, for some $\frac{1}{2} \leq \beta_{1} \leq \alpha \leq 1 ; h(z)$ defined by (1.1) and

$$
\gamma_{1}+1<\frac{\beta_{1}}{\alpha}
$$

Then, for every $z \in L$ and $P_{n} \in \wp_{n}, n \in \mathbb{N}$, there exists $c_{5}=c_{5}\left(L, p, \gamma_{1}, \beta_{1}, \alpha\right)>0$, such that

and

$$
\left|P_{n}(z)\right| \leq c_{5}\left(n^{s_{1}}+\left|z-z_{1}\right|^{\sigma_{1}} n^{1 / p \alpha}\right)\left\|P_{n}\right\|_{\mathcal{L}_{p}(h, L)},
$$

$$
\left|P_{n}\left(z_{1}\right)\right| \leq c_{6} n^{\frac{\gamma_{1}+1}{p \beta_{1}}}\left\|P_{n}\right\|_{\mathcal{L}_{p}(h, L)},
$$

where

$$
s_{1}=\frac{1+\gamma_{1}}{p \beta_{1}}, \sigma_{1}=\frac{\beta_{1}}{2 \alpha}-\frac{1+\gamma_{1}}{2} .
$$

Since $\alpha \geq \beta_{1}$, (2.7) will be satisfied when $-1<\gamma_{1}<0$. So, from (2.8) it can be seen that, the order of the height of $P_{n}$ in point $z_{1}$ and points $z \in L, z \neq z_{1}$, where $h(z) \rightarrow \infty$ and curve $L$ not having singularity, acts itself identically. Thus, the conditions (2.7) can be called "algebraic pole" conditions of the order $\lambda_{1}=1-\frac{\alpha}{\beta_{1}}\left(1+\gamma_{1}\right)$.

In case, if $L$ and $h(z)$ have of two singular points, it can be written as

$$
\begin{gathered}
\left|P_{n}(z)\right| \leq c_{7}\left(\left|z-z_{1}\right|^{\sigma_{1}} n^{s_{2}}+\left|z-z_{2}\right|^{\sigma_{2}} n^{s_{1}}\right. \\
\left.+\left|z-z_{1}\right|^{\sigma_{1}}\left|z-z_{2}\right|^{\sigma_{2}} n^{1 / p \alpha}\right)\left\|P_{n}\right\|_{\mathcal{L}_{p}(h, L)}, z \in L,
\end{gathered}
$$

where $s_{i}, \sigma_{i}, i=1,2$, defined as in (2.10), for $i=1$ and analogously for $i=2$, respectively.

Theorem 2.8 is also correct if the curve $L$ has at point $z_{1}$ algebraic pole and at points $\left\{z_{k}\right\}, k \geq 2$, singularity, in which satisfying the interference conditions (2.4).

Corollary 2.9. If $L \in C\left(1, \lambda, \nu_{1}\right)$, then $L \in \widetilde{Q}_{\alpha, \beta_{1}}$ for $\alpha=1$ (2.3) and $\beta_{1}=\frac{1}{\nu_{1}}$ [15]. Consequently, if the condition

$$
\left(\gamma_{1}+1\right) \nu_{1}<1
$$

satisfies on the point $z_{1}$, then, for $p=2$, from (2.8) and (2.10), we have

$$
\left|P_{n}(z)\right| \leq\left(c_{6} n^{s_{1}}+c_{7}\left|z-z_{1}\right|^{\sigma_{1}} \sqrt{n}\right)\left\|P_{n}\right\|_{\mathcal{L}_{2}(h, L)},
$$


where

$$
s_{1}=\frac{1}{2}\left(1+\gamma_{1}\right) \nu_{1}, \sigma_{1}=\frac{1}{2}\left(\frac{1}{\nu_{1}}-1-\gamma_{1}\right) .
$$

For $P_{n} \equiv Q_{n}$, estimation (2.12) coincides from the result by P.K. Suetin in [28, Theorem 2]. Therefore, the Theorem 2.8 generalizes the result in [28, Th2] for $1 \leq \nu_{1} \leq 2$ and extends this result to the more general curves of the complex plane.

\section{Some auxiliary results}

For $a>0$ and $b>0$, we will use the notations " $a \preceq b$ " (order inequality), if $a \leq c b$ and " $a \asymp b$ " are equivalent to $c_{1} a \leq b \leq c_{2} a$ for some constants $c, c_{1}, c_{2}$ (independent of $a$ and $b$ ), respectively.

The following definitions of the $K$-quasiconformal curves are well-known (see, for example, [9], [14, p.97] and [24]).

Definition 3.1. The Jordan arc (or curve) $L$ is called $K$-quasiconformal $(K \geq 1)$, if there is a $K$-quasiconformal mapping $f$ of the region $D \supset L$ in such that $f(L)$ is a line segment (or circle).

Let $F(L)$ denotes the set of all sense preserving plane homeomorphisms $f$ of the region $D \supset L$ such that $f(L)$ is a line segment (or circle) and lets define

$$
K_{L}:=\inf \{K(f): f \in F(L)\},
$$

where $K(f)$ is the maximal dilatation of a such mapping $f . L$ is a quasiconformal curve, if $K_{L}<\infty$, and $L$ is a $K$-quasiconformal curve, if $K_{L} \leq K$.

Remark 3.2. It is well-known that if we are not interested in the coefficients of quasiconformality of the curve, then the definitions of "quasicircle" and "quasiconformal curve" are identical whereas, if we are also interested in the coefficients of quasiconformality of the given curve, then a consideration is taken if the curve $L$ is $K$-quasiconformal; therefore, it is $\kappa$-quasicircle with $\kappa=\frac{K^{2}-1}{K^{2}+1}$.

Following of the Remark 3.2, the both terms will be used for simplicity, depending on the situation.

For $z \in \mathbb{C}$ and $M \subset \mathbb{C}$, we set $\rho(z, M)=\operatorname{dist}(z, M):=\inf \{|z-\zeta|: \zeta \in M\}$.

Lemma 3.3. [3] Let $L$ be a $K$-quasiconformal curve, $z_{1} \in L, z_{2}, z_{3} \in \Omega \cap\left\{z:\left|z-z_{1}\right| \preceq\right.$ $\left.\rho\left(z_{1}, L_{r_{0}}\right)\right\} ; w_{j}=\Phi\left(z_{j}\right), j=1,2,3$. Then,

a) The statements $\left|z_{1}-z_{2}\right| \preceq\left|z_{1}-z_{3}\right|$ and $\left|w_{1}-w_{2}\right| \preceq\left|w_{1}-w_{3}\right|$ are equivalent. So statements $\left|z_{1}-z_{2}\right| \asymp\left|z_{1}-z_{3}\right|$ and $\left|w_{1}-w_{2}\right| \asymp\left|w_{1}-w_{3}\right|$ also are equivalent.

b) If $\left|z_{1}-z_{2}\right| \preceq\left|z_{1}-z_{3}\right|$, then

$$
\left|\frac{w_{1}-w_{3}}{w_{1}-w_{2}}\right|^{\varepsilon} \preceq\left|\frac{z_{1}-z_{3}}{z_{1}-z_{2}}\right| \preceq\left|\frac{w_{1}-w_{3}}{w_{1}-w_{2}}\right|^{c}
$$

where $\varepsilon<1, c>1,0<r_{0}<1$ are the constants depending on $G$ and $L_{r_{0}}:=$ $\left\{z=\psi(w):|w|=r_{0}\right\}$.

Lemma 3.4. Let $G \in Q(\kappa)$ for some $0 \leq \kappa<1$. Then,

$$
\left|\Psi\left(w_{1}\right)-\Psi\left(w_{2}\right)\right| \succeq\left|w_{1}-w_{2}\right|^{1+\kappa},
$$

for all $w_{1}, w_{2} \in \bar{\Delta}$.

This fact follows from [21, p.287, Lemma 9.9] and estimation for the $\Psi^{\prime}$ (see, for example, [11, Th.2.8]):

$$
\left|\Psi^{\prime}(\tau)\right| \asymp \frac{\rho(\Psi(\tau), L)}{|\tau|-1} .
$$

Let $\left\{z_{j}\right\}_{j=1}^{m}$ be the fixed system of the points on $L$ and the weight function $h(z)$ defined as (1.1). 
Lemma 3.5. [8] Let $L$ be a rectifiable Jordan curve and $h(z)$ is defined as in (1.1). Then, for arbitrary $P_{n}(z) \in \wp_{n}$, any $R>1$ and $n \in \mathbb{N}$, we have

$$
\left\|P_{n}\right\|_{\mathcal{L}_{p}\left(h, L_{R}\right)} \leq R^{n+\frac{1+\gamma *}{p}}\left\|P_{n}\right\|_{\mathcal{L}_{p}(h, L)}, p>0 .
$$

Remark 3.6. In case of $h(z) \equiv 1$, the estimation (3.2) has been proved in [12].

\section{Proofs of theorems}

\subsection{Proof of Theorem 2.5}

Proof. Suppose that $L \in \widetilde{Q}_{\alpha}$, for some $\frac{1}{2} \leq \alpha \leq 1, i=\overline{1, m}$ be given and $h(z)$ is defined as in (1.1). Let $w=\varphi_{R}(z)$ be the univalent conformal mapping of $G_{R}, R>1$, onto the $B$, normalized by $\varphi_{R}(0)=0, \varphi_{R}^{\prime}(0)>0$, and let $\left\{\xi_{j}\right\}, 1 \leq j \leq m \leq n$, be zeros of $P_{n}(z)$ lying on $G_{R}$. Let

$$
B_{m, R}(z):=\prod_{j=1}^{m} B_{j, R}(z)=\prod_{j=1}^{m} \frac{\varphi_{R}(z)-\varphi_{R}\left(\xi_{j}\right)}{1-\overline{\varphi_{R}\left(\xi_{j}\right)} \varphi_{R}(z)},
$$

denotes the Blashke function with respect to zeros $\left\{\xi_{j}\right\}, 1 \leq j \leq m \leq n$, of $P_{n}(z)$. Clearly,

$$
\left|B_{m, R}(z)\right| \equiv 1, z \in L_{R},
$$

and

$$
\left|B_{m, R}(z)\right|<1, z \in G_{R}
$$

For any $p>0$ and $z \in G_{R}$, let us set:

$$
T_{n}(z):=\left[\frac{P_{n}(z)}{B_{m, R}(z)}\right]^{p / 2} .
$$

The function $T_{n}(z)$ is analytic in $G_{R}$ and continuous on $\bar{G}_{R}$, not having zeros in $G_{R}$. We take an arbitrary continuous branch of the $T_{n}(z)$ and we maintain the same designation for this branch. Then, the Cauchy integral representation for the $T_{n}(z)$ in $G_{R}$ gives

$$
T_{n}(z)=\frac{1}{2 \pi i} \int_{L_{R}} T_{n}(\zeta) \frac{d \zeta}{\zeta-z}, z \in G_{R},
$$

and then

$$
T_{n}\left(z_{j}\right)=\frac{1}{2 \pi i} \int_{L_{R}} T_{n}(\zeta) \frac{d \zeta}{\zeta-z_{j}} .
$$

Now, let $z \in L$. Multiplying the numerator and the denominator of the integrand by $h^{1 / 2}(\zeta)$, according to the Hölder inequality, from (4.2) and (4.3), we obtain:

$$
\begin{aligned}
& \left|P_{n}\left(z_{j}\right)\right| \leq\left(\frac{1}{2 \pi}\right)^{\frac{2}{p}}\left(\int_{L_{R}} h(\zeta)\left|P_{n}(\zeta)\right|^{p}|d \zeta|\right)^{1 / p} \\
& \times\left(\int_{L_{R}} \frac{|d \zeta|}{\prod_{j=1}^{m}\left|\zeta-z_{j}\right|^{2+\gamma_{j}}}\right)^{1 / p}=:\left(\frac{1}{2 \pi}\right)^{\frac{2}{p}} J_{n, 1} \times J_{n, 2},
\end{aligned}
$$

where

$$
J_{n, 1}:=\left\|P_{n}\right\|_{\mathcal{L}_{p}\left(h, L_{R}\right)}, J_{n, 2}:=\left(\int_{L_{R}} \frac{|d \zeta|}{\prod_{j=1}^{m}\left|\zeta-z_{j}\right|^{2+\gamma_{j}}}\right)^{1 / p} .
$$


Then, from Lemma 3.5, for the each points $\left\{z_{j}\right\}_{j=1}^{m} \in L$, we have:

$$
\left|P_{n}\left(z_{j}\right)\right| \preceq\left\|P_{n}\right\|_{\mathcal{L}_{p}} \cdot\left(J_{n, 2}\right)^{1 / p} .
$$

To estimate the integral $J_{n, 2}$, we introduce

$$
w_{j}:=\Phi\left(z_{j}\right), \varphi_{j}:=\arg w_{j}, L^{j}:=L \cap \bar{\Omega}^{j}, \quad L_{R}^{j}:=L_{R} \cap \bar{\Omega}^{j}, j=\overline{1, m},
$$

where $\Omega^{j}:=\Psi\left(\Delta_{j}^{\prime}\right)$

$$
\begin{aligned}
\Delta_{1}^{\prime}: & =\left\{t=R e^{i \theta}: R>1, \frac{\varphi_{m}+\varphi_{1}}{2} \leq \theta<\frac{\varphi_{1}+\varphi_{2}}{2}\right\}, \\
\Delta_{m}^{\prime} & :=\left\{t=R e^{i \theta}: R>1, \frac{\varphi_{m-1}+\varphi_{m}}{2} \leq \theta<\frac{\varphi_{m}+\varphi_{1}}{2}\right\},
\end{aligned}
$$

and, for $j=\overline{2, m-1}$

$$
\Delta_{j}^{\prime}:=\left\{t=R e^{i \theta}: R>1, \frac{\varphi_{j-1}+\varphi_{j}}{2} \leq \theta<\frac{\varphi_{j}+\varphi_{j+1}}{2}\right\} .
$$

Since the points $\left\{z_{j}\right\}_{j=1}^{m} \in L$ are distinct, we get:

$$
\left(J_{n, 2}\right)^{p}=\sum_{i=1}^{m} \int_{L_{R}^{i}} \frac{|d \zeta|}{\prod_{j=1}^{m}\left|\zeta-z_{j}\right|^{2+\gamma_{j}}} \asymp \sum_{i=1}^{m} \int_{L_{R}^{i}} \frac{|d \zeta|}{\left|\zeta-z_{i}\right|^{2+\gamma_{i}}}=: \sum_{i=1}^{m} J_{n, 2}^{i},
$$

where

$$
J_{n, 2}^{i}:=\int_{L_{R}^{i}} \frac{|d \zeta|}{\left|\zeta-z_{i}\right|^{2+\gamma_{i}}}, i=\overline{1, m} .
$$

It remains to estimate the integrals $J_{n, 2}^{i}$ for each $i=\overline{1, m}$. For the simplicity of our next calculations, we assume that

$$
m=1 ; \quad R=1+\frac{\varepsilon_{0}}{n} .
$$

Let the numbers $\delta_{1}, \delta_{1}^{*}, 0<\delta_{1}<\delta_{1}^{*}<\delta_{0}<\operatorname{diam} \bar{G}$, are chosen from the Definition 2.6. By denoting

$$
l_{R, 1}^{1}:=L_{R}^{1} \cap \Omega\left(z_{1}, \delta_{1}\right), l_{R, 2}^{1}:=L_{R}^{1} \backslash l_{R, 1}^{1}, F_{R, i}^{1}:=\Phi\left(l_{R, i}^{1}\right), i=1,2,
$$

from (4.9), we get

$$
J_{n, 2}^{1}:=\int_{L_{R}^{1}} \frac{|d \zeta|}{\left|\zeta-z_{1}\right|^{2+\gamma_{1}}}=\int_{l_{R, 1}^{1}} \frac{|d \zeta|}{\left|\zeta-z_{1}\right|^{2+\gamma_{1}}}+\int_{l_{R, 2}^{1}} \frac{|d \zeta|}{\left|\zeta-z_{1}\right|^{2+\gamma_{1}}} .
$$

Then, by applying Lemma 3.3, we have:

$$
\begin{aligned}
& \int_{l_{R, 1}^{1}} \frac{|d \zeta|}{\left|\zeta-z_{1}\right|^{2+\gamma_{1}}}=\int_{\Phi\left(l_{R, 1}^{1}\right)} \frac{\rho(\Psi(\tau), L)|d \tau|}{\left|\Psi(\tau)-\Psi\left(w_{1}\right)\right|^{2+\gamma_{1}}(|\tau|-1)} \\
& \preceq \int_{\Phi\left(l_{R, 1}^{1}\right)} \frac{|d \tau|}{\left|\Psi(\tau)-\Psi\left(w_{1}\right)\right|^{1+\gamma_{1}}(|\tau|-1)} \\
& \preceq n \int_{\Phi\left(l_{R, 1}^{1}\right)} \frac{|d \tau|}{\left|\tau-w_{1}\right|^{\frac{\gamma_{1}+1}{\alpha}} \preceq n^{\frac{\gamma_{1}+1}{\alpha}},} \\
& \int_{l_{R, 2}^{1}} \frac{|d \zeta|}{\left|\zeta-z_{1}\right|^{2+\gamma_{1}}} \preceq\left(\delta_{1}\right)^{2+\gamma_{1}} m e s l_{R, 1}^{1} \preceq 1 .
\end{aligned}
$$


Then from (4.11)-(4.13), we get:

$$
J_{n, 2}^{1} \preceq n^{\frac{\gamma_{1}+1}{\alpha}} .
$$

By combining (4.7)-(4.14), we obtain:

$$
\left|P_{n}\left(z_{1}\right)\right| \preceq n^{\frac{\gamma_{1}+1}{p \alpha}}\left\|P_{n}\right\|_{\mathcal{L}_{p}},
$$

and, according to (4.10), the proof is completed.

\subsection{Proof of Theorem 2.8}

Proof. Suppose that $L \in \widetilde{Q}_{\alpha, \beta_{1}}$, for some $\frac{1}{2} \leq \beta_{1} \leq \alpha \leq 1$, be given and $h(z)$ defined as in (1.1). Let the functions $B_{m, R}(z)$ and $T_{R}(z)$ be constructed as in the beginning to the proof of Theorem 2.5 by (4.1)- (4.4). By taking the arbitrary fixed branch of $T_{R}(z)$, for any $z \in G_{R}$ from (4.5), we get:

$$
\begin{aligned}
T_{n}(z)-T_{n}\left(z_{1}\right) & =\frac{1}{2 \pi i} \int_{L_{R}} T_{n}(\zeta)\left[\frac{1}{\zeta-z}-\frac{1}{\zeta-z_{1}}\right] d \zeta, \\
& =\frac{1}{2 \pi i} \int_{L_{R}}\left[\frac{P_{n}(\zeta)}{B_{m, R}(\zeta)}\right]^{p / 2}\left[\frac{z-z_{1}}{(\zeta-z)\left(\zeta-z_{1}\right)}\right] d \zeta .
\end{aligned}
$$

For arbitrary $z \in L, z \neq z_{1}$, multiplying both sides of the equality by $\left(z-z_{1}\right)^{-\sigma_{1}}$, we obtain:

$$
\left|\frac{T_{n}(z)-T_{n}\left(z_{1}\right)}{\left(z-z_{1}\right)^{\sigma_{1}}}\right| \leq \frac{1}{2 \pi} \int_{L_{R}}\left|P_{n}(\zeta)\right|^{p / 2}\left|\frac{\left(z-z_{1}\right)^{1-\sigma_{1}}}{(\zeta-z)\left(\zeta-z_{1}\right)}\right||d \zeta|,
$$

since $\left|B_{m, R}(\zeta)\right|=1$, for $\zeta \in L_{R}$. By multiplying the numerator and the denominator of the integrand via $h^{1 / 2}(\zeta)$ and by applying the Hölder inequality, we obtain:

$$
\begin{gathered}
\left|\frac{T_{n}(z)-T_{n}\left(z_{1}\right)}{\left(z-z_{1}\right)^{\sigma_{1}}}\right| \leq \frac{1}{2 \pi}\left(\int_{L_{R}} h(\zeta)\left|P_{n}(\zeta)\right|^{p}|d \zeta|\right)^{1 / 2} \\
\times\left(\int_{L_{R}} \frac{\left|z-z_{1}\right|^{2-2 \sigma_{1}}}{|\zeta-z|^{2}\left|\zeta-z_{1}\right|^{2+\gamma_{1}}}|d \zeta|\right)^{1 / 2}=: \frac{1}{2 \pi}\left(J_{n, 1} \times J_{n, 2}\right)^{1 / 2},
\end{gathered}
$$

where

$$
\begin{aligned}
& J_{n, 1}:=\int_{L_{R}} h(\zeta)\left|P_{n}(\zeta)\right|^{p}|d \zeta|=\left\|P_{n}\right\|_{\mathcal{L}_{p}\left(h, L_{R}\right)}^{p}, \\
& J_{n, 2}:=\int_{L_{R}} \frac{\left|z-z_{1}\right|^{2-2 \sigma_{1}}}{|\zeta-z|^{2}\left|\zeta-z_{1}\right|^{2+\gamma_{1}}}|d \zeta| .
\end{aligned}
$$

Then, for $z \in L$, from Lemma 3.5, we have:

$$
\left|\frac{T_{n}(z)-T_{n}\left(z_{1}\right)}{\left(z-z_{1}\right)^{\sigma_{1}}}\right| \preceq\left\|P_{n}\right\|_{\mathcal{L}_{p}}^{\frac{p}{2}} \cdot\left(J_{n, 2}\right)^{1 / 2}, z \in L \backslash\left\{z_{1}\right\} .
$$

From (4.4), we obtain:

$$
\left|\frac{P_{n}(z)}{B_{m, R}(z)}\right|^{p / 2} \leq c_{9}\left|\frac{P_{n}\left(z_{1}\right)}{B_{m, R}\left(z_{1}\right)}\right|^{p / 2}+c_{10}\left\|P_{n}\right\|_{\mathcal{L}_{p}}^{\frac{p}{2}} \cdot\left(J_{n, 2}\right)^{1 / 2} .
$$


According to well-known inequalities [29, p.121]

$$
\begin{aligned}
& |A+B|^{p} \leq 2^{p-1}\left(|A|^{p}+|B|^{p}\right), p>1, \\
& |A+B|^{p} \leq|A|^{p}+|B|^{p}, 0<p \leq 1, A>0, B>0,
\end{aligned}
$$

from (4.16), we obtain:

$$
\left|P_{n}(z)\right| \leq c_{11}\left|\frac{B_{m, R}(z)}{B_{m, R}\left(z_{1}\right)}\right|\left|P_{n}\left(z_{1}\right)\right|+c_{12}\left\|P_{n}\right\|_{\mathcal{L}_{p}} \cdot\left(J_{n, 2}\right)^{1 / p} .
$$

Since $\left|B_{m, R}(z)\right|<1$, for $z \in L$ and $\left|B_{m, R}(\zeta)\right|=1$, for $\zeta \in L_{R}$, then there exists $\varepsilon_{1}$, where $0<\varepsilon_{1}<1$, in such that the following is fulfilled:

$$
\left|B_{m, R}\left(z_{1}\right)\right|>1-\varepsilon_{1} .
$$

Then, from (4.18) and (4.19), for each $z \in L \backslash\left\{z_{1}\right\}$, we have

$$
\left|P_{n}(z)\right| \leq c_{13}\left|P_{n}\left(z_{1}\right)\right|+c_{12}\left\|P_{n}\right\|_{\mathcal{L}_{p}} \cdot\left(J_{n, 2}\right)^{1 / p}, p>0 .
$$

By the Theorem 2.5, the estimation for $\left|P_{n}\left(z_{1}\right)\right|$ is known.Therefore, finding of an estimation of $J_{n, 2}$

$$
J_{n, 2}=\int_{L_{R}} \frac{\left|z-z_{1}\right|^{2-2 \sigma_{1}}}{|\zeta-z|^{2}\left|\zeta-z_{1}\right|^{2+\gamma_{1}}}|d \zeta| .
$$

is needed for completion. We set:

$$
\begin{aligned}
L_{R, 1}^{1}: & =L_{R}^{1} \cap \Omega\left(z_{1}, \delta_{1}\right), L_{R, 2}^{1}:=L_{R}^{1} \cap\left(\Omega\left(z_{1}, \delta_{1}^{*}\right) \backslash \Omega\left(z_{1}, \delta_{1}\right)\right), \\
L_{R, 3}^{1}: & =L_{R} \backslash\left(L_{R, 1}^{1} \cup L_{R, 2}^{1}\right) ; \quad F_{R, j}^{1}:=\Phi\left(L_{R, j}^{1}\right), \\
L_{1}^{1}: & =L^{1} \cap D\left(z_{1}, \delta_{1}\right), L_{2}^{1}:=L^{1} \cap\left(D\left(z_{1}, \delta_{1}^{*}\right) \backslash D\left(z_{1}, \delta_{1}\right)\right), \\
L_{3}^{1} & :=L \backslash\left(L_{1}^{1} \cup L_{2}^{1}\right) ; \quad F_{i}^{1}:=\Phi\left(L_{i}^{1}\right), i=1,2,3,
\end{aligned}
$$

then, from (4.15), we get:

$$
J_{n, 2}=\sum_{i=1}^{3} J_{n, 2}\left(L_{R, i}^{1}\right)
$$

where

$$
J_{n, 2}(l):=\int_{l} \frac{\left|z-z_{1}\right|^{2-2 \sigma_{1}}}{|\zeta-z|^{2}\left|\zeta-z_{1}\right|^{2+\gamma_{1}}}|d \zeta|,
$$

for $l \subset L$. There are two possible cases: the point $z$ may lie on $L^{1}$ or $L^{2}$. Suppose first, $z$ $\in L^{1}$. If $z \in L_{i}^{1}$, then $w \in F_{i}^{1}$, for $i=1,2,3$. Consider the individual cases:

1) Let $z \in L_{1}^{1}$.

1.1) By applying (4.17), we have:

$$
\begin{aligned}
J_{n, 2}\left(L_{R, 1}^{1}\right) & =\int_{L_{R, 1}^{1}} \frac{\left|z-z_{1}\right|^{2-2 \sigma_{1}}}{|\zeta-z|^{2}\left|\zeta-z_{1}\right|^{2+\gamma_{1}}}|d \zeta| \\
& \preceq \int_{L_{R, 1}^{1}} \frac{\left[|\zeta-z|+\left|\zeta-z_{1}\right|\right]^{2-2 \sigma_{1}}}{|\zeta-z|^{2}\left|\zeta-z_{1}\right|^{2+\gamma_{1}}}|d \zeta| \\
& =\int_{L_{R, 1}^{1}} \frac{|d \zeta|}{|\zeta-z|^{2 \sigma_{1}}\left|\zeta-z_{1}\right|^{2+\gamma_{1}}}+\int_{L_{R, 1}^{1}} \frac{|d \zeta|}{|\zeta-z|^{2}\left|\zeta-z_{1}\right|^{2 \sigma_{1}+\gamma_{1}}} .
\end{aligned}
$$

Lets

$$
\begin{aligned}
& L_{R, j}^{1,1}:=\left\{\zeta \in L_{R, j}^{1}:\left|\zeta-z_{1}\right| \geq|\zeta-z|\right\}, \\
& L_{R, j}^{1,2}:=L_{R, j}^{1} \backslash L_{R, j}^{1,1}, \quad F_{R, j}^{1, i}:=\Phi\left(\begin{array}{l}
1, i \\
R, j
\end{array}\right), i, j=1,2 .
\end{aligned}
$$


Then, from (4.23), we get:

$$
\begin{aligned}
& J_{n, 2}\left(L_{R, 1}^{1}\right) \preceq \int_{L_{R, 2}^{1,1}} \frac{|d \zeta|}{|\zeta-z|^{2 \sigma_{1}+2+\gamma_{1}}}+\int_{L_{R, 2}^{1,2}} \frac{|d \zeta|}{\left|\zeta-z_{1}\right|^{2 \sigma_{1}+2+\gamma_{1}}} \\
& \preceq \int_{F_{R, 2}^{1,1}} \frac{\rho(\Psi(\tau), L)|d \tau|}{|\Psi(\tau)-\Psi(w)|^{2 \sigma_{1}+2+\gamma_{1}}(|\tau|-1)} \\
& +\int_{F_{R, 2}^{1,2}} \frac{\rho(\Psi(\tau), L)|d \tau|}{|\Psi(\tau)-\Psi(w)|^{2 \sigma_{1}+2+\gamma_{1}}(|\tau|-1)} \\
& \preceq n \int_{F_{R, 1}^{1,1}} \frac{|d \tau|}{|\tau-w|^{\frac{2 \sigma_{1}+1+\gamma_{1}}{\beta_{1}}}}+n \int_{F_{R, 1}^{1,2}} \frac{|d \tau|}{|\tau-w|^{\frac{2 \sigma_{1}+1+\gamma_{1}}{\beta_{1}}}} \\
& \preceq n \cdot n^{\frac{2 \sigma_{1}+1+\gamma_{1}}{\beta_{1}}-1}+n \cdot n^{\frac{2 \sigma_{1}+1+\gamma_{1}}{\beta_{1}}-1} \preceq n^{\frac{2 \sigma_{1}+1+\gamma_{1}}{\beta_{1}}} .
\end{aligned}
$$

1.2) For any $\zeta \in L_{R, 2}^{1}$ and $z \in L_{1}^{1},\left|\zeta-z_{1}\right| \geq \delta_{1}$ and by Definition 2.6, we obtain:

$$
\begin{aligned}
|\zeta-z|^{\alpha} & \succeq \max \left\{\left|\zeta-z_{1}\right|^{\alpha-\beta_{1}} ;\left|z-z_{1}\right|^{\alpha-\beta_{1}}\right\}|w-\tau| \\
& =\left|\zeta-z_{1}\right|^{\alpha-\beta_{1}}|w-\tau| \geq \delta_{1}^{\alpha-\beta_{1}}|w-\tau| \succeq|w-\tau| .
\end{aligned}
$$

Then, for this case we get:

$$
\begin{aligned}
& J_{n, 2}\left(L_{R, 2}^{1}\right)=\int_{L_{R, 2}^{1}} \frac{\left|z-z_{1}\right|^{2-2 \sigma_{1}}}{|\zeta-z|^{2}\left|\zeta-z_{1}\right|^{2+\gamma_{1}}}|d \zeta| \\
& \preceq \int_{L_{R, 2}^{1}} \frac{\left[|\zeta-z|+\left|\zeta-z_{1}\right|\right]^{2-2 \sigma_{1}}}{|\zeta-z|^{2}\left|\zeta-z_{1}\right|^{2+\gamma_{1}}}|d \zeta| \\
& =\int_{L_{R, 2}^{1}} \frac{|d \zeta|}{|\zeta-z|^{2 \sigma_{1}}\left|\zeta-z_{1}\right|^{2+\gamma_{1}}}+\int_{L_{R, 2}^{1}} \frac{|d \zeta|}{|\zeta-z|^{2}\left|\zeta-z_{1}\right|^{2 \sigma_{1}+\gamma_{1}}} \\
& \leq \frac{1}{\delta_{1}^{2+\gamma_{1}}} \int_{L_{R, 2}^{1}} \frac{|d \zeta|}{|\zeta-z|^{2 \sigma_{1}}}+\frac{1}{\delta_{1}^{2 \sigma_{1}+\gamma_{1}}} \int_{L_{R, 2}^{1}} \frac{|d \zeta|}{|\zeta-z|^{2}} \\
& \preceq \int_{F_{R, 2}^{1}} \frac{\rho(\Psi(\tau), L)|d \tau|}{|\Psi(\tau)-\Psi(w)|^{2 \sigma_{1}}(|\tau|-1)} \\
& +\int_{F_{R, 2}^{1}} \frac{\rho(\Psi(\tau), L)|d \tau|}{|\Psi(\tau)-\Psi(w)|^{2}(|\tau|-1)} \\
& \preceq n \int_{F_{R, 1}^{1,1}} \frac{|d \tau|}{|\tau-w|^{\frac{2 \sigma_{1}-1}{\alpha}}}+n \int_{F_{R, 1}^{1,2}} \frac{|d \tau|}{|\tau-w|^{\frac{1}{\alpha}}} \\
& \preceq n \cdot n^{\frac{2 \sigma_{1}-1}{\alpha}-1}+n \cdot n^{\frac{1}{\alpha}-1} \preceq n^{\frac{1}{\alpha}} .
\end{aligned}
$$


1.3) For any $\zeta \in L_{R, 3}^{1}$ and $z \in L_{1}^{1}, \quad\left|\zeta-z_{1}\right| \geq \delta_{1}^{*}$ and $|\zeta-z| \geq \delta_{1}^{*}-\delta_{1}$. Then, we have

$$
\begin{aligned}
J_{n, 2}\left(L_{R, 3}^{1}\right) & =\int_{L_{R, 3}^{1}} \frac{\left|z-z_{1}\right|^{2-2 \sigma_{1}}}{|\zeta-z|^{2}\left|\zeta-z_{1}\right|^{2+\gamma_{1}}}|d \zeta| \\
& \leq \frac{\left(\operatorname{diam} \bar{G}_{R}\right)^{2-2 \sigma_{1}} m e s L}{\left(\delta_{1}^{*}-\delta_{1}\right)^{2-2 \sigma_{1}}\left(\delta_{1}^{*}\right)^{2}} \preceq 1 .
\end{aligned}
$$

2) Let $z \in L_{2}^{1}$.

2.1) According to $\left|\zeta-z_{1}\right|<\left|z-z_{1}\right|$, by Definition 2.6, we obtain:

$$
\begin{aligned}
|\zeta-z|^{\alpha} & \succeq \max \left\{\left|\zeta-z_{1}\right|^{\alpha-\beta_{1}} ;\left|z-z_{1}\right|^{\alpha-\beta_{1}}\right\}|w-\tau| \\
& =\left|z-z_{1}\right|^{\alpha-\beta_{1}}|w-\tau| \geq \delta_{1}^{\alpha-\beta_{1}}|w-\tau| \succeq|w-\tau| .
\end{aligned}
$$

Then, we get:

$$
\begin{aligned}
& J_{n, 2}\left(L_{R, 1}^{1}\right)=\int_{L_{R, 1}^{1}} \frac{\left|z-z_{1}\right|^{2-2 \sigma_{1}}}{|\zeta-z|^{2}\left|\zeta-z_{1}\right|^{2+\gamma_{1}}}|d \zeta| \\
& \preceq \int_{L_{R, 1}^{1}} \frac{\left[|\zeta-z|+\left|\zeta-z_{1}\right|\right]^{2-2 \sigma_{1}}}{|\zeta-z|^{2}\left|\zeta-z_{1}\right|^{2+\gamma_{1}}}|d \zeta| \\
& =\int_{L_{R, 1}^{1}} \frac{|d \zeta|}{|\zeta-z|^{2 \sigma_{1}}\left|\zeta-z_{1}\right|^{2+\gamma_{1}}}+\int_{L_{R, 1}^{1}} \frac{|d \zeta|}{|\zeta-z|^{2}\left|\zeta-z_{1}\right|^{2 \sigma_{1}+\gamma_{1}}} \\
& \preceq \int_{L_{R, 1}^{1,1}} \frac{|d \zeta|}{|\zeta-z|^{2 \sigma_{1}+2+\gamma_{1}}}+\int_{L_{R, 1}^{1,2}} \frac{|d \zeta|}{\left|\zeta-z_{1}\right|^{2 \sigma_{1}+2+\gamma_{1}}} \\
& \preceq \int_{F_{R, 2}^{1,1}} \frac{\rho(\Psi(\tau), L)|d \tau|}{|\Psi(\tau)-\Psi(w)|^{2 \sigma_{1}+2+\gamma_{1}}(|\tau|-1)}+\int_{F_{R, 2}^{1,2}} \frac{\rho(\Psi(\tau), L)|d \tau|}{\left|\Psi(\tau)-\Psi\left(w_{1}\right)\right|^{2 \sigma_{1}+2+\gamma_{1}}(|\tau|-1)} \\
& \preceq n \int_{F_{R, 1}^{1,1}} \frac{|d \tau|}{|\tau-w|^{\frac{2 \sigma_{1}+1+\gamma_{1}}{\alpha}}}+n \int_{F_{R, 1}^{1,2}} \frac{|d \tau|}{\left|\tau-w_{1}\right|^{\frac{2 \sigma_{1}+1+\gamma_{1}}{\beta_{1}}}} \\
& \preceq n \cdot n^{\frac{2 \sigma_{1}+1+\gamma_{1}}{\alpha}-1}+n \cdot n^{\frac{2 \sigma_{1}+1+\gamma_{1}}{\beta_{1}}-1} \preceq n^{\frac{2 \sigma_{1}+1+\gamma_{1}}{\beta_{1}}} .
\end{aligned}
$$

Therefore, in this case we have

$$
J_{n, 2}\left(L_{R, 1}^{1}\right) \preceq n^{\frac{2 \sigma_{1}+1+\gamma_{1}}{\beta_{1}}} .
$$

2.2) For any $\zeta \in L_{R, 2}^{1}$ and $z \in L_{2}^{1},\left|\zeta-z_{1}\right| \geq \delta_{1}$ and analogously to the case 1.2), in this case from Definition 2.6, we obtain

$$
\begin{aligned}
|\zeta-z|^{\alpha} & \succeq \max \left\{\left|\zeta-z_{1}\right|^{\alpha-\beta_{1}} ;\left|z-z_{1}\right|^{\alpha-\beta_{1}}\right\}|w-\tau| \\
& \geq \delta_{1}^{\alpha-\beta_{1}}|w-\tau| \succeq|w-\tau|
\end{aligned}
$$

and then,

$$
J_{n, 2}\left(L_{R, 2}^{1}\right)=\int_{L_{R, 1}^{1}} \frac{\left|z-z_{1}\right|^{2-2 \sigma_{1}}}{|\zeta-z|^{2}\left|\zeta-z_{1}\right|^{2+\gamma_{1}}}|d \zeta|
$$


The uniform and pointwise estimates for polynomials...

$$
\begin{aligned}
& \preceq \frac{\left(\delta_{1}^{*}\right)^{2-2 \sigma_{1}}}{\left(\delta_{1}\right)^{2+\gamma_{1}}} \int_{L_{R, 1}^{1}} \frac{|d \zeta|}{|\zeta-z|^{2}} \\
& \preceq \int_{F_{R, 2}^{1}} \frac{\rho(\Psi(\tau), L)|d \tau|}{|\Psi(\tau)-\Psi(w)|^{2}(|\tau|-1)} \preceq n \int_{F_{R, 2}^{1}} \frac{|d \tau|}{|\Psi(\tau)-\Psi(w)|} \\
& \preceq n \int_{F_{R, 2}^{1}} \frac{|d \tau|}{|\tau-w|^{\frac{1}{\alpha}}} \preceq n^{\frac{1}{\alpha}} .
\end{aligned}
$$

2.3) For any $\zeta \in L_{R, 3}^{1}$ and $z \in L_{2}^{1},\left|\zeta-z_{1}\right| \geq \delta_{1}^{*}$ and $|\zeta-z| \geq \delta_{1}^{*}-\delta_{1}$. Then, we have:

$$
\begin{aligned}
& J_{n, 2}\left(L_{R, 3}^{1}\right)=\int_{L_{R, 3}^{1}} \frac{\left|z-z_{1}\right|^{2-2 \sigma_{1}}}{|\zeta-z|^{2}\left|\zeta-z_{1}\right|^{2+\gamma_{1}}}|d \zeta| \\
\preceq & \frac{\left(\delta_{1}^{*}\right)^{2-2 \sigma_{1}}}{\left(\delta_{1}\right)^{2+\gamma}} \int_{L_{R, 3}^{1}} \frac{|d \zeta|}{|\zeta-z|^{2}} \preceq \int_{L_{R, 3}^{1}} \frac{|d \zeta|}{|\zeta-z|^{2}} \\
\preceq & \int_{F_{R, 3}^{1}} \frac{\rho(\Psi(\tau), L)|d \tau|}{|\Psi(\tau)-\Psi(w)|^{2}(|\tau|-1)} \preceq n \int_{F_{R, 3}^{1}} \frac{|d \tau|}{|\Psi(\tau)-\Psi(w)|} \\
\preceq & n \int_{F_{R, 3}^{1}} \frac{|d \tau|}{|\tau-w|^{\frac{1}{\alpha}}} \preceq n^{\frac{1}{\alpha}} .
\end{aligned}
$$

3) Let $z \in L_{3}^{1}$.

3.1) Analogously to previous cases, we get:

$$
\begin{aligned}
& J_{n, 2}\left(L_{R, 1}^{1}\right)=\int_{L_{R, 1}^{1}} \frac{\left|z-z_{1}\right|^{2-2 \sigma_{1}}}{|\zeta-z|^{2}\left|\zeta-z_{1}\right|^{2+\gamma_{1}}}|d \zeta| \\
& \quad \leq \frac{(2 \operatorname{diam} \bar{G})^{2-2 \sigma_{1}}}{\left(\delta_{1}^{*}-\delta_{1}\right)^{2}} \int_{L_{R, 1}^{1}} \frac{|d \zeta|}{\left|\zeta-z_{1}\right|^{2+\gamma_{1}}} \\
& \preceq \int_{F_{R, 1}^{1}} \frac{\rho(\Psi(\tau), L)|d \tau|}{|\Psi(\tau)-\Psi(w)|^{2+\gamma_{1}}(|\tau|-1)} \\
& \preceq n \int_{F_{R, 1}^{1}} \frac{|d \tau|}{|\tau-w|^{\frac{2+\gamma_{1}}{\beta_{1}}} \preceq n^{\frac{2+\gamma_{1}}{\beta_{1}}} .}
\end{aligned}
$$

3.2) Since, from Definition 2.6,

$$
\begin{aligned}
|\zeta-z|^{\alpha} & \succeq \max \left\{\left|\zeta-z_{1}\right|^{\alpha-\beta_{1}} ;\left|z-z_{1}\right|^{\alpha-\beta_{1}}\right\}|w-\tau| \\
& \geq \delta_{1}^{\alpha-\beta_{1}}\left|w^{\prime}-\tau\right| \succeq|w-\tau|,
\end{aligned}
$$

then, we have:

$$
J_{n, 2}\left(L_{R, 2}^{1}\right)=\int_{L_{R, 2}^{1}} \frac{\left|z-z_{1}\right|^{2-2 \sigma_{1}}}{|\zeta-z|^{2}\left|\zeta-z_{1}\right|^{2+\gamma_{1}}}|d \zeta|
$$




$$
\begin{aligned}
& \leq \frac{\left(\delta_{1}^{*}\right)^{2-2 \sigma_{1}}}{\left(\delta_{1}\right)^{2+\gamma_{1}}} \int_{L_{R, 2}^{1}} \frac{|d \zeta|}{|\zeta-z|^{2}} \\
& \preceq \int_{F_{R, 2}^{1}} \frac{\rho(\Psi(\tau), L)|d \tau|}{|\Psi(\tau)-\Psi(w)|^{2}(|\tau|-1)} \preceq n \int_{F_{R, 2}^{1}} \frac{|d \tau|}{|\Psi(\tau)-\Psi(w)|} \\
& \preceq n \int_{F_{R, 2}^{1}} \frac{|d \tau|}{|\tau-w|^{\frac{1}{\alpha}}} \preceq n^{\frac{1}{\alpha}} .
\end{aligned}
$$

3.3) Also, we get:

$$
\begin{aligned}
& J_{n, 2}\left(L_{R, 3}^{1}\right)=\int_{L_{R, 3}^{1}} \frac{\left|z-z_{1}\right|^{2-2 \sigma_{1}}}{|\zeta-z|^{2}\left|\zeta-z_{1}\right|^{2+\gamma_{1}}}|d \zeta| \\
\leq & \left(\delta_{1}^{*}\right)^{-2 \sigma_{1}-\gamma_{l}} \int_{L_{R, 3}^{1}} \frac{|d \zeta|}{|\zeta-z|^{2}} \\
\preceq & \int_{F_{R, 3}^{1}} \frac{\rho(\Psi(\tau), L)|d \tau|}{|\Psi(\tau)-\Psi(w)|^{2}(|\tau|-1)} \preceq n \int_{F_{R, 3}^{1}} \frac{|d \tau|}{|\Psi(\tau)-\Psi(w)|} \\
\preceq & n \int_{F_{R, 3}^{1}} \frac{|d \tau|}{|\tau-w|^{\frac{1}{\alpha}}} \preceq n^{\frac{1}{\alpha}} .
\end{aligned}
$$

By combining the estimations of (4.18), (4.20)-(4.32), finally we obtain:

$$
\left|P_{n}(z)\right| \leq c_{13}\left|P_{n}\left(z_{1}\right)\right|+c_{12}\left\|P_{n}\right\|_{\mathcal{L}_{p}} \cdot n^{\frac{1}{\alpha p}}, p>0 .
$$

and, then the proof of (2.8) is completed.

The estimation of (2.9) follows from the Theorem 2.5 for $\alpha=\beta_{1}$.

Acknowledgment. This work is supported by TUBITAK under Project No: 115F652.

\section{References}

[1] F.G. Abdullayev, On the some properties on orthogonal polynomials over the regions of complex plane 1. Ukr. Math. J. 52 (12), 1807-1817, 2000.

[2] F.G. Abdullayev, On the interference of the weight boundary contour orthogonal polynomials over the region, J. of Comp. Anal. and Appl. 6 (1), 31-42, 2004.

[3] F.G. Abdullayev and V.V. Andrievskii, On the orthogonal polynomials in the domains with K-quasiconformal boundary, Izv. Akad. Nauk Azerb. SSR., Ser. FTM, 1, 3-7, 1983.

[4] F.G. Abdullayev and C.D. Gün, On the behavior of the algebraic polynomials in regions with piecewise smooth boundary without cusps, Ann. Polon. Math. 111, 39-58, 2014.

[5] F.G. Abdullayev and N.P. Özkartepe, On the Behavior of the Algebraic Polynomial in Unbounded Regions with Piecewise Dini -Smooth Boundary, Ukr. Math. J., 66 (5), 579-597, 2014.

[6] F.G. Abdullayev and N.P. Özkartepe, On the growth of algebraic polynomials in the whole complex plane, J. Korean Math. Soc. 52 (4) 699-725, 2015.

[7] F.G. Abdullayev and N.P. Özkartepe, Uniform and pointwise polynomial inequalities in regions with cusps in the weighted Lebesgue space, Jaen J. Approx. 7 (2), 231-261, 2015. 
[8] F.G. Abdullayev, N.P. Özkartepe, C.D. Gün, Uniform and pointwise polynomial inequalities in regions without cusps in the weighted Lebesgue space, Bulletin of Tbilisi ICMC 18 (1), 146-167, 2014.

[9] L. Ahlfors, Lectures on Quasiconformal Mappings, Princeton, NJ: Van Nostrand, 1966.

[10] V.V. Andrievskii, Weighted Polynomial Inequalities in the Complex Plane, J. Approx. Theory 164(9), 1165-1183, 2012.

[11] V.V. Andrievskii, V.I. Belyi, and V.K. Dzyadyk, Conformal invariants in constructive theory of functions of complex plane, Atlanta: World Federation Publ.Com., 1995.

[12] E. Hille, G. Szegö, and J.D. Tamarkin, On some generalization of a theorem of $A$. Markoff, Duke Math. 3, 729-739, 1937.

[13] D. Jackson, Certain problems on closest approximations, Bull. Amer. Math. Soc. 39, 889-906, 1933.

[14] O. Lehto and K.I. Virtanen, Quasiconformal Mapping in the Plane, Springer Verlag, Berlin, 1973.

[15] F.D. Lesley, Hölder continuity of conformal mappings at the boundary via the strip method, Indiana Univ. Math. J. 31, 341-354, 1982.

[16] D.I. Mamedhanov, Inequalities of S.M.Nikol'skii type for polynomials in the complex variable on curves, Soviet Math. Dokl. 15, 34-37, 1974.

[17] D.I. Mamedhanov, On Nikol'skii-type inequalities with new characteristic, Dokl. Math. 82, 882-883, 2010.

[18] G.V. Milovanovic, D.S. Mitrinovic, and Th.M. Rassias, Topics in Polynomials: Extremal Problems, Inequalities, Zeros, World Scientific, Singapore, 1994.

[19] S.M. Nikol'skii, Approximation of Function of Several Variable and Imbeding Theorems, Springer-Verlag, New-York, 1975.

[20] N.P. Özkartepe and F.G. Abdullayev, Interference of the weight and boundary contour for algebraic polynomials in the weighted Lebesgue spaces I, Ukr. Math. J. 68(10), 1574-1590, 2017.

[21] Ch. Pommerenke, Univalent Functions, Göttingen, Vandenhoeck \& Ruprecht, 1975.

[22] Ch. Pommerenke, Boundary Behavior of Conformal Maps, Springer-Verlag, Berlin, 1992.

[23] I. Pritsker, Comparing Norms of Polynomials in One and Several Variables, J. Math. Anal. Appl. 216, 685-695, 1997.

[24] S. Rickman, Characterisation of quasiconformal arcs, Ann. Acad. Sci. Fenn., Ser. A, Mathematica 395, 30 p, 1966.

[25] G. Szegö and A. Zigmund, On certain mean values of polynomials, J. Anal. Math. 3, 225-244, 1954.

[26] P.K. Suetin, The ordinally comparison of various norms of polynomials in the complex domain, Matematicheskie zapiski Uralskogo Gos. Universiteta 5 (4), 1966 (in Russian).

[27] P.K. Suetin, Main properties of the orthogonal polynomials along a circle, Uspekhi Math. Nauk 21 (2 (128)), 41-88, 1966.

[28] P.K. Suetin, On some estimates of the orthogonal polynomials with singularities weight and contour, Sib. Math. J VIII (3), 1070-1078, 1967 (in Russian).

[29] J.L. Walsh, Interpolation and Approximation by Rational Functions in the Complex Domain, AMS, 1960.

[30] S.E. Warschawski, On differentiability at the boundary in conformal mapping, Proc. Amer. Math. Soc., 12, 614-620, 1961. 